Article

\title{
Improving the Method of Measuring the Electron Density via the Asymmetry of Hydrogenic Spectral Lines in Plasmas by Allowing for Penetrating Ions
}

\author{
Paul Sanders $\mathbb{D}^{\mathbb{D}}$ and Eugene Oks * \\ Physics Department, 206 Allison Lab, Auburn University, Auburn, AL 36849, USA; phs0007@auburn.edu \\ * Correspondence: goks@physics.auburn.edu
}

Received: 9 March 2018; Accepted: 8 April 2018; Published: 18 April 2018

check for updates

\begin{abstract}
There was previously proposed and experimentally implemented a new diagnostic method for measuring the electron density $\mathrm{N}_{\mathrm{e}}$ using the asymmetry of hydrogenic spectral lines in dense plasmas. Compared to the traditional method of deducing $\mathrm{N}_{\mathrm{e}}$ from the experimental widths of spectral lines, the new method has the following advantages. First, the traditional method requires measuring widths of at least two spectral lines (to isolate the Stark broadening from competing broadening mechanisms), while for the new diagnostic method it is sufficient to obtain the experimental profile of just one spectral line. Second, the traditional method would be difficult to implement if the center of the spectral lines was optically thick, while the new diagnostic method could still be used even in this case. In the theory underlying this new diagnostic method, the contribution of plasma ions to the spectral line asymmetry was calculated only for configurations where the perturbing ions were outside the bound electron cloud of the radiating atom/ion (non-penetrating configurations). In the present paper, we take into account the contribution to the spectral line asymmetry from penetrating configurations, where the perturbing ion is inside the bound electron cloud of the radiating atom/ion. We show that in high-density plasmas, the allowance for penetrating ions can result in significant corrections to the electron density deduced from the spectral line asymmetry.
\end{abstract}

Keywords: asymmetry of spectral lines; penetrating ions; spectroscopic diagnostics of plasmas; electron density measurements

\section{Introduction}

In medium-density plasmas, profiles of hydrogenic spectral lines look symmetric, but in high-density plasmas, they become asymmetric. This asymmetry is caused primarily by the nonuniformity of the ion microfield, as noted by Sholin and his co-workers in papers [1-3] -for the latest advances in the theory of the asymmetry we refer to papers $[4,5]$ and the references therein, of which we especially note papers [6,7]. (There are also secondary sources of the asymmetry, as discussed in more detail below in the first paragraph of Section 2). Often, the blue maximum of the spectral line is higher than the red maximum, and the positions of the intensity maxima are asymmetrical with respect to the unperturbed line center.

A new diagnostic method for measuring the electron density using the asymmetry of hydrogenic spectral lines in dense plasmas was proposed and implemented in paper [8]. In that paper, in particular, from the experimental asymmetry of the C VI Lyman-delta line emitted by a vacuum spark discharge, the electron density was deduced to be $\mathrm{N}_{\mathrm{e}}=3 \times 10^{20} \mathrm{~cm}^{-3}$. This value of $\mathrm{N}_{\mathrm{e}}$ was in good agreement with the electron density determined from the experimental widths of C VI Lyman-beta and Lyman-delta lines. 
Later, this diagnostic method was also employed in the experiment presented in paper [9]. In that laser-induced breakdown spectroscopy experiment, the electron density $\mathrm{N}_{\mathrm{e}} \sim 3 \times 10^{17} \mathrm{~cm}^{-3}$ was determined from the experimental asymmetry of the $\mathrm{H}$ I Balmer-beta (H-beta) line.

This new diagnostic method has the following advantages compared to the method of deducing $\mathrm{N}_{\mathrm{e}}$ from the experimental widths of spectral lines. First, the latter, traditional, method requires measuring widths of at least two spectral lines-because the widths are affected not only by the Stark broadening, but also by competing broadening mechanisms, such as, e.g., Doppler broadening. In distinction, to use the new diagnostic method, it is sufficient to obtain the experimental profile of just one spectral line-because the Doppler broadening does not cause the asymmetry.

Second, the traditional method based on experimental widths would be difficult to implement if the center of the spectral lines were optically thick. In distinction, the new diagnostic method can still be used even if the spectral line is optically thick in its central part. This is because the overwhelming contribution to the asymmetry originates from the wings of the spectral line, the wings usually being optically thin. More details can be found in Section 1.6 of [10] ${ }^{1}$.

In the theory underlying this new diagnostic method, the contribution of plasma ions to the spectral line asymmetry was calculated only for configurations where the perturbing ions were outside the "atomic sphere", i.e., outside the bound electron cloud of the radiating atom/ion (non-penetrating configurations). In the present paper, we take into the contribution to the spectral line asymmetry from penetrating configurations, i.e., from configurations where the perturbing ion is inside the bound electron cloud of the radiating atom/ion (hereafter, radiator). We show that, in high-density plasmas, the allowance for penetrating ions can result in significant corrections to the electron density deduced from the spectral line asymmetry.

\section{Allowance for Penetrating Ions}

Let us first present a brief overview of the underlying theory for non-penetrating configurations. The dipole interaction of the radiator with perturbing ions outside the bound electron cloud, being calculated in the first order of the perturbation theory, splits the spectral line into Stark components symmetrically with respect to the unperturbed frequency or wavelength-in terms of both positions and intensities of the Stark components. The quadrupole interactions of the radiator with perturbing ions outside the bound electron cloud, being calculated in its first nonvanishing order, causes the asymmetry of the Stark splitting-in terms of both positions and intensities of the Stark components. The latter is the primary source of the asymmetry: other sources of the asymmetry-such as, but not limited to, e.g., the dipole interaction in the second order (known as the quadratic Stark effect), the quadrupole interaction in the second order, the octupole interaction in the first order-add to the asymmetry only higher-order corrections in terms of the corresponding small parameter $\mathrm{n}^{2} \mathrm{a}_{0} \mathrm{~N}_{\mathrm{e}}^{1 / 3} / \mathrm{Z}^{4 / 3}$, where $\mathrm{n}$ is the principal quantum number of the upper level involved in the radiative transition, $a_{0}$ is the Bohr radius, $Z$ is the charge of plasma ions and the nuclear charge of the radiating ion. ${ }^{2}$

1 We note that Ref. [40] from chp. 1 of [10] on the paper referred to here as [8] has typographic errors. The correct one is our Ref. [8] here.

2 The Boltzmann factor $\exp (-\hbar \Delta \omega / \mathrm{T})$ also contributes to the asymmetry (here $\Delta \omega$ is the detuning from the unperturbed frequency of the spectral line and $\mathrm{T}$ is the temperature). For quasistatic wings, $\hbar \Delta \omega / \mathrm{T}$ scales with the electron density as $\left(\mathrm{a}_{0} \mathrm{Ne}_{\mathrm{e}}{ }^{1 / 3}\right)^{2}$. Therefore, for plasmas of the electron densities $\mathrm{N}_{\mathrm{e}}<<6.7-10^{24} \mathrm{~cm}^{-3}$ (the right side being the atomic unit of the electron density), the Boltzmann factor contribution to the asymmetry is much smaller than the quadrupole interaction contribution to the asymmetry that scales as $\mathrm{a}_{0} \mathrm{Ne}_{\mathrm{e}}{ }^{1 / 3}$. Additionally, there is also the factor $\left(1+\omega / \omega_{0}\right)^{4}$ caused by the scaling of the dipole radiation intensity ( $\omega_{0}$ being the unperturbed frequency). The asymmetry contributions of this factor and of the Boltzmann factor essentially cancel each other out (see, e.g., Section 5.11 of paper [5]). (Continued at the bottom of the next page). There is also so-called trivial contribution to the asymmetry caused by the conversion from the frequency scale to the wavelength scale. This consists of two factors (see, e.g., Section 5.8 of paper [5]): the transformation of the argument $\Delta \omega$ (given by Equation (24) from [5]) and the transformation of the intensity (given by Equation (25) from [5]). These two factors essentially cancel each other out (as shown in [5]), so that the resulting trivial contribution to the asymmetry is much smaller than the quadrupole interaction contribution to the asymmetry. 
However, in paper [11], it was shown that the quadrupole interaction, despite causing the asymmetric splitting of the spectral line into Stark components, does not shift the center of gravity of the line profile. Therefore, in the new diagnostic method presented in paper [8], first the center of gravity of the experimental profile was determined, and then it was taken as the reference point. Then, with respect to this point, the integrated intensities of the blue $\left(I_{B}\right)$ and red $\left(I_{R}\right)$ wings of the experimental profile were found. After that, the experimental degree of asymmetry, defined as

$$
\rho_{\text {quad }}=\frac{I_{B}-I_{R}}{0.5\left[I_{B}+I_{R}\right]},
$$

was determined and then compared with the corresponding theoretical value given below.

The theoretical intensities of the blue and red wings, resulting from dipole and quadrupole interactions of the radiator with perturbing ions outside the bound electron cloud, can be expressed as follows (see paper [8]):

$$
I_{B}=\sum_{k>0} I_{k}^{(0)}\left(1+\frac{a_{o}}{Z_{r} R_{o}} \epsilon_{k}^{(1)}\left\langle\frac{R_{0}}{\mathrm{R}}\right\rangle\right)
$$

and

$$
I_{R}=\sum_{k<0} I_{k}^{(0)}\left(1+\frac{a_{o}}{Z_{r} R_{o}} \epsilon_{k}^{(1)}\left\langle\frac{R_{0}}{R}\right\rangle\right)
$$

where $Z_{p}$ is the charge of perturbing ions, $Z_{r}$ is the nuclear charge of the radiator, $a_{0}$ is the Bohr radius, and $R_{o}=\left[(4 \pi / 3) N_{\mathrm{p}}\right]^{-1 / 3}$ is the mean interionic distance, $N_{\mathrm{p}}=\mathrm{N}_{\mathrm{e}} / \mathrm{Z}_{\mathrm{p}}$ being the perturbing ion density. Here, $I_{k}^{(0)}$ and $\epsilon_{k}^{(1)}$ are the unperturbed intensity and the quadrupole correction to the intensity, respectively, the subscript $k$ being the label of Stark components of the spectral line; $k>0$ and $k<0$ correspond to the blue-shifted and red-shifted components, respectively (the values of $I_{k}^{(0)}$ and $\epsilon_{k}^{(1)}$ for several Lyman and Balmer lines were tabulated in paper [2]). The quantity $<R_{0} / R>$ is the scaled inverse distance between the perturbing ion and the radiator averaged over the distribution of such distances.

Finally, the theoretical degree of asymmetry was presented in paper [8] in the form:

$$
\rho_{\text {quad }}=0.46204\left(\frac{N_{e}\left[\mathrm{~cm}^{-3}\right]}{10^{21}}\right)^{\frac{1}{3}} \frac{1}{Z_{p}^{\frac{1}{3}} Z_{r}} \sum_{k>0} I_{k}^{(0)} \epsilon_{k}^{(1)},
$$

Then the electron density $\mathrm{N}_{\mathrm{e}}$ was determined in paper [8] by substituting the experimental degree of asymmetry into the left side of Equation (4).

In the present paper, we consider the contribution of penetrating ions to the spectral line asymmetry in order to refine this diagnostic method. For simplicity, we limit ourselves below to the practically important case $Z_{p}=Z_{r}=Z$. The energy shifts due to penetrating ions can be calculated by the perturbation theory on the basis of the spherical wave functions of the so-called "united atom" of the nuclear charge $2 \mathrm{Z}$.

The perturbed energy shifts (counted from the unperturbed energies) for the orbital quantum number $l>0$ are given by (see, e.g., Equations (6) and (7) from paper [12] or Equations (5.11) and (5.12) from book [13]):

$$
E_{n l m}=-\frac{8\left[1(1+1)-3 \mathrm{~m}^{2}\right] Z^{4} R^{2} e^{2}}{\mathrm{a}_{\mathrm{o}}{ }^{3} \mathrm{n}^{3} 1(1+1)(2 \mathrm{l}-1)(2 l+1)(2 \mathrm{l}+3)} .
$$

For the case of $l=0$, the calculated energy shift is:

$$
E_{n 00}=\frac{8 Z^{4} R^{2} e^{2}}{3 \mathrm{a}_{\mathrm{o}}^{3} n^{3}} .
$$


We note that Equation (6) can also be obtained from Equation (5), first by setting $\mathrm{m}=0$, and then by canceling out $l(l+1)$ in the numerator and denominator, and by setting $l=0$. (This was mentioned in book [12], but in Equation (5.11) from [12] corresponding to our Equation (6), there was a typographic error in the sign.)

The frequency change of an individual Stark component is thus given by

$$
\Delta \omega_{k}=-\frac{Z^{2} e^{2} \Delta_{k}^{1}}{2 \hbar \mathrm{a}_{\mathrm{o}}^{3}} R^{2}
$$

where

$$
\Delta_{k}^{1}=16 Z^{2}\left[\frac{l(l+1)-3 m^{2}}{n^{3} l(l+1)(2 l-1)(2 l+1)(2 l+3)}-\frac{l^{\prime\left(l^{\prime}+1\right)}-3 m^{\prime 2}}{n^{\prime 3} l^{\prime}\left(l^{\prime}+1\right)\left(2 l^{\prime}-1\right)\left(2 l^{\prime}+1\right)\left(2 l^{\prime}+3\right)}\right] .
$$

For the specific case where either $l=0$ or $l^{\prime}=0$, Equation (8) reduces to

$$
\Delta_{k}^{1}=\left\{\begin{array}{c}
16 Z^{2}\left[\frac{1}{3 n^{3}}-\frac{l^{\prime}\left(l^{\prime}+1\right)-3 m^{\prime 2}}{n^{\prime 3} l^{\prime}\left(l^{\prime}+1\right)\left(2 l^{\prime}-1\right)\left(2 l^{\prime}+1\right)\left(2 l^{\prime}+3\right)}\right], l=0 ; l^{\prime} \neq 0 \\
16 Z^{2}\left[\frac{l(l+1)-3 m^{2}}{n^{3} l(l+1)(2 l-1)(2 l+1)(2 l+3)}-\frac{1}{3 n^{\prime 3}}\right], l^{\prime}=0 ; l \neq 0
\end{array} .\right.
$$

Then, the quasi-static profile of each Stark component can be represented in the form:

$$
S_{k}(\Delta \lambda)=\int_{0}^{u_{\max }} W(u)\left[I_{k}^{(0)}+I_{k}^{(1)}\right] \delta\left(\Delta \lambda-\frac{Z^{2} e^{2} \Delta_{k}^{1} \lambda_{0}^{2}}{4 \pi c \hbar a_{o}{ }^{3}} u\right) d u
$$

Here, $u \equiv R^{2}$, and the probability of finding the perturbing ion a distance $u$ away from the radiating atom is taken to be the binary distribution. For simplifying the integration, we use the expansion of the distribution in powers $u / R_{0}{ }^{2}$ and keep the terms up to $\sim u^{2}$ :

$$
W(u) d u=\frac{3 \sqrt{u}}{2 R_{o}^{3}} \exp \left(-\frac{\sqrt{u}^{3}}{R_{o}^{3}}\right) d u \approx \frac{3 \sqrt{u}}{2 R_{o}^{3}}-\frac{3 u^{2}}{2 R_{o}^{6}}
$$

For the case of a hydrogenic radiator under the presence of a penetrating ion, the relative intensities of each line component can be best calculated analytically using the robust perturbation theory developed by Oks and Uzer [14]. A more detailed explanation of this procedure is outlined in Appendix A. The relative intensities of each component can be written as

$$
I_{k}=\Delta_{I k}^{0}+Z^{2} \Delta_{I k}^{1} u^{2}
$$

where $\Delta_{I k}^{0}$ and $\Delta_{I k}^{1}$ are tabulated in Appendix B for each component of the spectral line Balmer-alpha, considered here as an example. These coefficients represent corrections to the intensity of the line, calculated from the perturbation theory briefly mentioned above.

The upper limit $u_{\max }$ of the integration in Equation (10) should be the smallest of the following two "candidates". One candidate for $u_{\max }$ is the root mean square size of the bound electron cloud, which depends on the sublevel in consideration:

$$
r_{\mathrm{rms}}=\sqrt{\frac{n^{2}}{2 Z^{2}}\left[5 n^{2}+1-3 l(l+1)\right]} .
$$

The other candidate for $u_{\text {max }}$ is defined by the limit of the applicability of the perturbation theory. Of course, this would ensure that formally calculated corrections to the energy and intensities of the spectral line would remain relatively small. 
The allowance for penetrating ions shifts the center of gravity of the spectral line, as shown in paper [15]. (This is the only contribution to the shift of the center of gravity, since the dipole and quadrupole interactions of the radiator with perturbing ions outside the bound electron cloud do not shift the center of gravity, as shown in paper [11] and mentioned above). For the He II Balmer-alpha line, which we use as an example, the center of gravity shift due to penetrating ions was calculated analytically in paper [15] to be

$$
\Delta \lambda_{P I}(m \AA)=17 \frac{N_{e}\left(\mathrm{~cm}^{-3}\right)}{10^{17}} .
$$

So, with the allowance for penetrating ions, the reference point for calculating the integrated intensities of the blue and red wings must be shifted by the amount given by Equation (14).

After carrying out the integration in Equation (10), the profile reduces to

$$
S_{k}(\Delta \lambda)=\left(\frac{Z^{2} e^{2} \Delta_{k}^{1} \lambda_{0}^{2}}{4 \pi c \hbar a_{o}{ }^{3}}\right)^{-1} I_{k}\left(u_{0}\right)\left(\frac{3 u_{0}^{\frac{1}{2}}}{2 R_{o}^{3}}-\frac{3 u_{0}^{2}}{2 R_{o}^{6}}\right) \Theta\left[u_{\max } \frac{Z^{2} e^{2} \Delta_{k}^{1} \lambda_{0}^{2}}{4 \pi \hbar a_{o}{ }^{3}}-|\Delta \lambda|\right],
$$

where $\Theta[\ldots]$ is the Heaviside step function and $u_{0}$ is the root of the delta function, given by

$$
u_{0}=\frac{4 \pi c \hbar a_{o}{ }^{3}}{Z^{2} e^{2} \Delta_{k}^{1} \lambda_{0}^{2}} \Delta \lambda .
$$

Thus, for the contributions of the penetrating ions to the integrated intensities of the blue and read parts of the line profile, we get

$$
\mathrm{I}_{\mathrm{PI}, \mathrm{B}}=\sum_{k<0} \int_{-\Delta \lambda_{\max }}^{\Delta \lambda_{P I}} S_{k}(\Delta \lambda) d \Delta \lambda
$$

and

$$
\mathrm{I}_{\mathrm{PI}, \mathrm{R}}=\sum_{k>0} \int_{\Delta \lambda_{P I}}^{\Delta \lambda_{\max }} S_{k}(\Delta \lambda) d \Delta \lambda,
$$

respectively. Here

$$
\Delta \lambda_{\max }=u_{\max } \frac{Z^{2} e^{2} \Delta_{k}^{1} \lambda_{0}^{2}}{4 \pi c \hbar a_{0}{ }^{3}},
$$

which is obtained by equating to zero the argument of the Heaviside step function. Additionally, what is meant in Equations (17) and (18) by $k<0$ (or $k>0$ ) is the inclusion of only those components which involve corrections to the energy that are positive (or negative), implying blue-shifted (or red-shifted) components of the spectral line.

By combining the above result with the contribution of the quadrupole interaction (the interaction of the radiator with perturbing ions outside the bound electron cloud) to the integrated intensities of the blue and read parts of the profile, we obtain our final result for the degree of asymmetry

$$
\rho_{a c t}=\frac{I_{B}+\mathrm{I}_{\mathrm{PI}, \mathrm{B}}-I_{R}-\mathrm{I}_{\mathrm{PI}, \mathrm{R}}}{0.5\left[I_{B}+\mathrm{I}_{\mathrm{PI}, \mathrm{B}}+I_{R}+\mathrm{I}_{\mathrm{PI}, \mathrm{R}}\right]},
$$

where subscript act stands for actual—in distinction to $\rho_{\text {quad }}$.

The combination of Equations (4) and (20) connects the degree of asymmetry with the electron density $\mathrm{N}_{\mathrm{e}}$ and thus allows a more accurate determination of the electron density from the experimental asymmetry. We illustrate this below by the example of the He II Balmer-alpha line.

Table 1 presents the following quantities for the He II Balmer-alpha line at five different values of the actual electron density:

- the theoretical degree of asymmetry $\rho_{\text {act }}$ calculated with the allowance for penetrating ions,

- the theoretical degree of asymmetry $\rho_{\text {quad }}$ calculated without the allowance for penetrating ions, 
- $\quad$ the electron density $\mathrm{N}_{\mathrm{e}, \text { quad }}$ that would be deduced from the experimental asymmetry degree while disregarding the contribution of the penetrating ions,

- the relative error $\left|N_{e, q u a d}-N_{e, a c t}\right| / N_{e, a c t}$ in determining the electron density from the experimental asymmetry degree while disregarding the contribution of the penetrating ions.

Table 1. The relative error in determining the electron density $\mathrm{N}_{\mathrm{e}}$ from the experimental asymmetry degree while disregarding the contribution of the penetrating ions for the He II Balmer-alpha line. The physical quantities in Table 1 are explained in the text directly above Table 1.

\begin{tabular}{|c|c|c|c|c|}
\hline $\mathrm{N}_{\mathrm{e}, \text { act }} /\left(10^{18} \mathrm{~cm}^{-3}\right)$ & $\rho_{\text {act }}$ & $\rho_{\text {quad }}$ & $\mathrm{N}_{\mathrm{e}, \text { quad }} /\left(10^{18} \mathrm{~cm}^{-3}\right)$ & $\left|N_{e, q u a d}-N_{e, a c t}\right| / N_{e, a c t}$ \\
\hline 2 & 0.0925 & 0.0955 & 1.82 & $9.03 \%$ \\
\hline 4 & 0.114 & 0.120 & 3.42 & $14.5 \%$ \\
\hline 6 & 0.128 & 0.138 & 4.86 & $19.1 \%$ \\
\hline 8 & 0.139 & 0.152 & 6.16 & $23.1 \%$ \\
\hline 10 & 0.147 & 0.163 & 7.33 & $26.7 \%$ \\
\hline
\end{tabular}

It is seen that in high-density plasmas, the allowance for penetrating ions can indeed result in significant corrections to the electron density deduced from the spectral line asymmetry.

\section{Conclusions}

To improve the diagnostic method for measuring the electron density using the asymmetry of spectral lines in dense plasmas, we took into account the contribution to the spectral line asymmetry from penetrating configurations, i.e., from the configurations where the perturbing ion is inside the bound electron cloud of the radiating atom/ion. After performing the corresponding analytical calculations, we demonstrated that in high-density plasmas, the allowance for penetrating ions can result in significant corrections to the electron density deduced from the spectral line asymmetry.

It is worth clarifying why we took into account the shift of the line as a whole due to penetrating ions, but did not take into account other mechanisms shifting the line as a whole, such as, e.g., plasma polarization shift and the shift by plasma electrons. The experimental integrated intensities of the blue $\left(I_{B}\right)$ and red $\left(I_{R}\right)$ parts of the profile are calculated with respect to the experimental center of gravity of the profile. The latter shifts of the line as a whole do not contribute to the asymmetry, and thus should not affect the experimental values of $I_{B}$ and $I_{R}$. The reason why we took into account the shift of the line as a whole by penetrating ions is that penetrating ions contribute simultaneously to both the asymmetry and the shift of the line as the whole. Since these two effects of penetrating ions are two sides of the same coin, both of them should be taken into account.

We mention in passing that the potential transition of electrons into the quasistatic regime is practically irrelevant to the asymmetry. Indeed, as Demura and Sholin wrote in paper [3], for the quadrupole interaction $U \sim Q / R^{3}$, which controls the asymmetry of hydrogenic spectral lines, electrons can become quasistatic at the frequency detuning from the line center $\Delta \omega \sim \mathrm{v}_{\mathrm{Te}}{ }^{3 / 2} / \mathrm{Q}^{1 / 2}$, where $\mathrm{v}_{\mathrm{Te}}$ is the mean thermal velocity of plasma electrons-according to Holstein [16] (see also Sobelman book [17]). However, such detuning significantly exceeds the mean separation between spectral lines-both for the Lyman and Balmer series, as noted by Demura and Sholin [3], thus making the potential transition of electron into the quasistatic regime irrelevant to the problem of asymmetry of hydrogenic spectral lines. A similar conclusion was drawn also in paper [18].

Finally, we note that the electron densities $\mathrm{N}_{\mathrm{e}} \sim\left(10^{18}-10^{19}\right) \mathrm{cm}^{-3}$, which we used in the illustrative example of the He II Balmer-alpha line, are achievable in plasma spectroscopy. Examples include experiment [19], with a hydrogen plasma, and experiment [20], with a helium plasma.

Author Contributions: Both authors contributed equally to this work.

Conflicts of Interest: The authors declare no conflict of interests. 


\section{Appendix A. Details of Calculating Perturbed Matrix Elements}

The redistribution of intensities of Stark components, along with wavelength shifts due to the presence of perturbing ions, play a crucial role in determining the degree of asymmetry of the spectral line. These values have been tabulated according to the robust perturbation theory developed by Oks and Uzer [14] based on using the super-generalized Runge-Lenz vector derived by Kryukov and Oks [21]. Since the unperturbed system has an additional constant of the motion (namely the Runge-Lenz vector), the task of calculating the corrections to the state is simplified. The reason for this beneficial result is that the correction to the Runge-Lenz vector is non-degenerate with respect to the same states that are degenerate in the correction to the Hamiltonian. The mixing of the states is elucidated by the Runge-Lenz vector correction under the influence of the perturbing ion. Here are some details, formulas being presented in atomic units.

According to paper [21], for the problem of an electron in the field of two Coulomb centers of charges $Z_{1}$ and $Z_{2}$, the additional conserved quantity is the following projection of the super-generalized Runge-Lenz vector on the internuclear axis

$$
A_{\mathrm{z}}=\mathbf{p} \times \mathbf{L} \cdot \mathbf{e}_{\mathbf{z}}-L^{2} / R-Z_{1} z / r-Z_{2}(R-z) /|\mathbf{R}-\mathbf{r}|+Z_{2},
$$

where $\mathbf{p}, \mathbf{L}$, and $\mathbf{r}$ are the linear momentum, the angular momentum, and the radius-vector of the electron, respectively; $\mathbf{R}$ is the vector directed from charge $Z_{1}$ to charge $Z_{2}$. For the case where $R<<r$, the unperturbed part $\mathrm{A}_{\mathrm{z} 0}$ of the operator $A_{z}$ can be chosen as

$$
\mathrm{A}_{\mathrm{z} 0}=-\mathrm{L}^{2} / \mathrm{R},
$$

corresponding to the unperturbed Hamiltonian of the so-called "united atom" of the nuclear charge $\mathrm{Z}_{1}+\mathrm{Z}_{2}$ :

$$
\mathrm{H}_{0}=\mathrm{p}^{2} / 2-\left(\mathrm{Z}_{1}+\mathrm{Z}_{2}\right) / \mathrm{r}
$$

Operators $\mathrm{H}_{0}$ and $\mathrm{A}_{\mathrm{z} 0}$ have common eigenfunctions (the spherical eigenfunctions of the Coulomb problem). The spectrum of eigenvalues of the operator $\mathrm{H}_{0}$ is degenerate. Therefore, calculating corrections to the eigenfunctions of the operator $\mathrm{H}_{0}$ using the standard perturbation theory would require going to the 2 nd order of the degenerate perturbation theory, thus involving generally infinite summations (see, e.g., the textbook [22]).

In distinction, the spectrum of eigenvalues of the operator $\mathrm{A}_{\mathrm{z} 0}$ is nondegenerate (the eigenvalues being- $l(l+1) / \mathrm{R})$. Therefore, the corrections to the eigenfunctions can be easily calculated in the $1 \mathrm{st}$ order of the standard nondegenerate perturbation theory. The coefficients of the corresponding linear combinations of the unperturbed eigenfunctions are

$$
<\mathrm{nl} l^{\prime} \mathrm{m}\left|\left(\mathrm{A}_{\mathrm{z}}-\mathrm{A}_{\mathrm{z} 0}\right)\right| \mathrm{n} l \mathrm{~m}>/\left\{\left[l^{\prime}\left(l^{\prime}+1\right)-l(l+1)\right] / \mathrm{R}\right\}
$$

and do not involve infinite summations. This example is another illustration of the advantages of the robust perturbation theory developed in paper [14] over the standard perturbation theory.

In this way, by using the first nonvanishing term of the expansion of the operator $\left(A_{z}-A_{z 0}\right)$ in powers of $\mathrm{R}$, we obtained the following expression for the 1st order corrections to the eigenfunctions for the specific case of $Z_{1}=Z_{2}=Z$ :

$$
\Psi_{n l m}^{(1)}=\frac{5\left[\frac{\left(l_{>}^{2}-m^{2}\right)\left(n^{2}-l_{>}^{2}\right)}{\left(2 l_{>}+1\right)\left(2 l_{>}-1\right)}\right]^{\frac{1}{2}}}{n\left[l(l+1)-l^{\prime}\left(l^{\prime}-1\right)\right]} Z R \Psi_{n l^{\prime} m^{\prime}}^{(0)}
$$

where $l_{>}$denotes the greater value between $l$ and $l^{\prime}$. The selection rules are $l^{\prime}=l \pm 1$ and $m^{\prime}=m$. 
We note that in the opposite case, where $R>>r$, the unperturbed part $A_{z 1,0}$ of the operator $A_{z}$ can be chosen in the usual way

$$
\mathrm{A}_{\mathrm{z} 1,0}=\mathrm{zp}^{2}-\mathrm{p}_{\mathrm{z}}(\mathbf{r p})-\mathrm{Z}_{1} \mathrm{z} / \mathrm{r},
$$

where the notation (rp) stands for the scalar product (also known as the dot-product) of the operators $\mathbf{r}$ and $\mathbf{p}$. The corresponding unperturbed Hamiltonian is

$$
\mathrm{H}_{1,0}=\mathrm{p}^{2} / 2-\mathrm{Z}_{1} / \mathrm{r}
$$

The operator $A_{z 1,0}$ has a nondegenerate spectrum of eigenvalues equal to $q / n$, where $q=\left(n_{1}-n_{2}\right)$ is the difference of the parabolic quantum numbers. Therefore, the first nonvanishing corrections to the common eigenfunctions of the operators $\mathrm{H}_{1,0}$ and $\mathrm{A}_{\mathrm{z} 1,0}$ can be easily calculated in the 1 st order of the standard nondegenerate perturbation theory. The coefficients of the corresponding linear combinations of the unperturbed eigenfunctions are

$$
<\mathrm{nl} \mathrm{m}\left|\mathrm{L}^{2}\right| \mathrm{n} l \mathrm{~m}>/\left[\left(q^{\prime} / \mathrm{n}-q / \mathrm{n}\right) \mathrm{R}\right]
$$

where $\left|q^{\prime}-q\right|=2$, as follows from the selection rules.

In distinction, to obtain the same corrections to the eigenfunctions using the operator $\mathrm{H}_{1,0}$, whose spectrum of eigenvalues is degenerate, it would require going to the 2 nd order of the degenerate perturbation theory and dealing with its complications, as Sholin did in his paper [2].

We note in passing that we also applied the robust perturbation theory [14] to analytically calculating corrections to the eigenfunctions due to the quadrupole interaction with ions outside the atomic electron cloud (non-penetrating ions), and we obtained the same analytical results as in the Sholin paper [2], but in a much simpler way. We also note that in paper [23], some corrections were presented to the input data from the tables in the Sholin paper [2].

\section{Appendix B. Table of Intensities and Energy Level Corrections for the He II Balmer-alpha line}

The perturbed intensity and frequency corrections for He II Balmer-alpha line are presented below. The quantum numbers of the upper and lower sublevels are in the spherical quantization.

Table A1. Corrections to the intensity and frequency of the He II Balmer-alpha line components.

\begin{tabular}{ccccc}
\hline Upper Sublevel & Lower Sublevel & $\Delta_{I k}^{0}$ & $\Delta_{I k}^{1}$ & $\Delta_{k}^{1}$ \\
\hline 322 & 211 & $\frac{768}{4715}$ & $\mathbf{0}$ & $\frac{173}{5670}$ \\
\hline 321 & 211 & $\frac{384}{4715}$ & $-\frac{32}{14145}$ & $\frac{197}{5670}$ \\
\hline 321 & 210 & $\frac{34}{4715}$ & $-\frac{64}{2829}$ & $-\frac{37}{567}$ \\
\hline 321 & 200 & $\mathbf{0}$ & $\frac{2792}{127305}$ & $\frac{949}{2835}$ \\
\hline 320 & 211 & $\frac{128}{4715}$ & $-\frac{128}{127305}$ & $\frac{41}{1134}$ \\
\hline 320 & 210 & $\frac{512}{4715}$ & $-\frac{3968}{127305}$ & $-\frac{181}{2835}$ \\
\hline 320 & 200 & $\mathbf{0}$ & $\frac{1168}{381915}$ & $\frac{953}{2835}$ \\
\hline 311 & 211 & $\mathbf{0}$ & $\frac{32}{14145}$ & $\frac{19}{810}$ \\
\hline 311 & 210 & $\mathbf{0}$ & $\frac{232}{14145}$ & $-\frac{31}{405}$ \\
\hline 311 & 200 & $\frac{160}{2829}$ & $-\frac{400}{25461}$ & $\frac{131}{405}$ \\
\hline 310 & 211 & $\mathbf{0}$ & $\frac{8}{3105}$ & $\frac{43}{810}$ \\
\hline 310 & 210 & $\mathbf{0}$ & $\frac{2512}{127305}$ & $-\frac{19}{405}$ \\
\hline 310 & 200 & $\frac{160}{2829}$ & $-\frac{280}{8487}$ & $\frac{143}{405}$ \\
\hline 300 & $\frac{5}{943}$ & $-\frac{40}{25461}$ & $-\frac{53}{810}$ \\
\hline 300 & 211 & $\frac{5}{943}$ & $-\frac{295}{101844}$ & $-\frac{67}{405}$ \\
\hline 300 & 210 & $\mathbf{0}$ & $\frac{5525}{305532}$ & $\frac{19}{81}$ \\
\hline
\end{tabular}




\section{References}

1. Kudrin, L.P.; Sholin, G.V. Asymmetry of spectral lines of hydrogen in plasma. Sov. Phys. Dokl. 1963, 7, 1015.

2. Sholin, G.V. On the nature of the asymmetry of the spectral line profiles of hydrogen in a dense plasma. Opt. Spectrosc. 1969, 26, 275.

3. Demura, A.V.; Sholin, G.V. Theory of the asymmetry of hydrogen-line Stark profiles in a dense plasma. J. Quant. Spectrosc. Radiat. Transf. 1975, 15, 881-899.

4. Djurovic, S.; Ćirišan, M.; Demura, A.V.; Demchenko, G.V.; Nikolić, D.; Gigosos, M.A.; González, M.A. Measurements of $\mathrm{H}_{\beta}$ Stark central asymmetry and its analysis through standard theory and computer simulations. Phys. Rev. E 2009, 79, 046402. [CrossRef] [PubMed]

5. Demura, A.V.; Demchenko, G.V.; Nikolic, D. Multiparametric dependence of hydrogen Stark profiles asymmetry. Eur. Phys. J. D 2008, 46, 111-127. [CrossRef]

6. Halenka, J.Z. Asymmetry of hydrogen lines in plasmas utilizing a statistical description of ion-quadruple interaction in Mozer-Baranger limit. Physics D 1990, 16, 1. [CrossRef]

7. Gunter, S.; Konies, A. Shifts and asymmetry parameters of hydrogen Balmer lines in dense plasmas. Phys. Rev. E 1997, 55, 907. [CrossRef]

8. Podder, N.K.; Clothiaux, E.J.; Oks, E. A method for density measurements employing an asymmetry of lineshapes in dense plasmas and its implementation in a vacuum spark discharge. J. Quant. Spectrosc. Radiat. Transf. 2000, 65, 441-453. [CrossRef]

9. Parigger, C.G.; Swafford, L.D.; Woods, A.C.; Surmick, D.M.; Witte, M.J. Asymmetric hydrogen beta electron density diagnostics of laser-induced plasma. Spectrochim. Acta Part B 2014, 99, 28-33. [CrossRef]

10. Oks, E. Diagnostics of Laboratory and Astrophysical Plasmas Using Spectral Lineshapes of One-, Two-, and Three-Electron Systems; World Scientific: Hackensack, NJ, USA; Singapore, 2017.

11. Oks, E. New type of shift of hydrogen and hydrogenlike spectral lines. J. Quant. Spectrosc. Radiat. Transf. 1997, 58, 821. [CrossRef]

12. Gavrilenko, V.P.; Oks, E.; Radchik, A.V. Hydrogen-like atom in the field of high-frequency linearly polarized electromagnetic radiation. Opt. Spectrosc. 1985, 59, 411.

13. Komarov, I.V.; Ponomarev, L.I.; Yu, S. Slavyanov, Spheroidal and Coulomb Spheroidal Functions; Nauka: Moscow, Russia, 1976. (In Russian)

14. Oks, E.; Uzer, T. A robust perturbation theory for degenerate states based on exact constants of the motion. Europhys. Lett. 2000, 49, 5. [CrossRef]

15. Sanders, P.; Oks, E. Estimate of the Stark Shift by Penetrating Ions within the Nearest Perturber Approximation for Hydrogenlike Spectral Lines in Plasmas. J. Phys. B 2017, 50, 245002. [CrossRef]

16. Holstein, T. Pressure broadening of spectral lines. Phys. Rev. 1950, 79, 744. [CrossRef]

17. Sobelman, I.I. An Introduction to the Theory of Atomic Spectra; Elsevier: Amsterdam, The Netherlands, 1972.

18. Oks, E.; Sholin, G.V. Boundary determination for electron quasistationarity using the asymmetry of the wings of hydrogen spectral line profiles. Opt. Spectrosc. 1972, 33, 217-218.

19. Kielkopf, J.F.; Allard, N.F. Shift and width of the Balmer series $\mathrm{H} \alpha$ line at high electron density in a laser-produced plasma. J. Phys. B At. Mol. Opt. Phys. 2014, 47, 155701. [CrossRef]

20. Marangos, J.P.; Burgess, D.D.; Baldwin, K.G.H. He II Balmer series line shifts in a dense z-pinch plasma. J. Phys. B At. Mol. Opt. Phys. 1988, 21, 3357. [CrossRef]

21. Kryukov, N.; Oks, E. Supergeneralized Runge-Lenz vector in the problem of two Coulomb or Newton centers. Phys. Rev. A 2012, 85, 054503. [CrossRef]

22. Landau, L.D.; Lifshitz, E.M. Quantum Mechanics; Pergamon: Oxford, UK, 1965.

23. Sanders, P.; Oks, E. Correcting the Input Data for Calculating the Asymmetry of Hydrogenic Spectral Lines in Plasmas. Atoms 2018, 6, 9. [CrossRef]

(c) 2018 by the authors. Licensee MDPI, Basel, Switzerland. This article is an open access article distributed under the terms and conditions of the Creative Commons Attribution (CC BY) license (http://creativecommons.org/licenses/by/4.0/). 